\title{
Particle Filter for Joint Blind Carrier Frequency Offset Estimation and Data Detection
}

\author{
Ali A. Nasir, Salman Durrani and Rodney A. Kennedy \\ School of Engineering, CECS, The Australian National University, Canberra, Australia. \\ Email: \{ali.nasir, salman.durrani, rodney.kennedy\}@ anu.edu.au
}

\begin{abstract}
This paper proposes a new blind algorithm for joint carrier offset estimation and data detection, which is based on particle filtering and recursively estimates the joint posterior probability density function of the unknown transmitted data and the unknown carrier offset. We develop new guidelines for resampling of the particles to take into account carrier offset estimation ambiguity at the edges of the range, and for fine tuning estimates to achieve fast, accurate convergence. The Mean Square Error (MSE) and Bit Error Rate (BER) performance of the proposed algorithm is studied through computer simulations. The results show that the proposed algorithm achieves fast convergence for the full acquisition range for normalized carrier frequency offsets.
\end{abstract}

Index Terms-Synchronization, Particle filters, Frequency offset estimation, Blind Algorithms.

\section{INTRODUCTION}

Carrier Frequency Offset (CFO) correction is a fundamental requirement for reliable data transmission in any wireless communication system. The CFOs arise because the transmitter and receiver oscillators can never be perfectly synchronous. The CFO manifests itself as a time-varying phase offset and results in a time-varying rotation of the data symbols. It is well known that even small residual errors in CFO estimation can lead to large errors in data detection [1]. Thus CFO must be compensated for accurately prior to data detection.

The existing CFO estimators can be classified as either data-aided or blind. Data aided schemes use training or pilot symbols (known to both the transmitter and the receiver) and are bandwidth consuming [2]. Blind schemes do not require any initial training and can be broadly classified into two categories: (a) algorithms which operate in feedback mode and employ Phase Lock Loop (PLL) or automatic frequency control loops [3]; and (b) algorithms which operate in feed forward mode and are based on open loop frequency estimation [4]-[6]. PLL based algorithms require a long convergence time and suffer from the well known $M$-fold symmetric ambiguity, resulting in a limited acquisition range for the frequency offset, i.e., $|f|<1 /(2 M)$, where $M$ is the constellation size and $f$ is the normalised digital frequency offset. Feed forward algorithms rely on the statistics of the received signal and require oversampling and long data records for processing.

In recent years, there has been growing interest in application of particle filtering (also known as Sequential Monte Carlo) methods to synchronization problem in wireless communication systems [7], [8]. Particle filters are a powerful tool for estimating unknown parameters in non-linear, nonGaussian, real-time applications. The core principle of particle filtering is to build a recursive Bayesian filter in order to estimate the posterior probability density function of the unknown parameters. Prior work in this area has looked at particle filters for joint blind equalization and data detection [9] and joint blind timing estimation and data detection [10], [11], respectively. To the best of author's knowledge, the use of particle filters for carrier frequency offset estimation has not been considered before.

In this paper, we propose a new particle filter based blind algorithm for joint carrier offset estimation and data detection in Additive White Gaussian Noise (AWGN) channels. The proposed algorithm recursively estimates the joint posterior probability density function of the unknown transmitted data and the unknown carrier offsets and achieves very fast carrier recovery. The major contributions of this paper, in comparison to previous research, are as follows:

- We propose a particle filters based algorithm for non-data aided, joint estimation of frequency offset and transmitted symbols. We develop new guidelines for resampling of the particles to taken into account carrier offset estimation ambiguity at the edges of the frequency range and for fine tuning of estimates.

- The results demonstrate that the proposed algorithm achieves full-range acquisition of carrier frequency offset $|f|<1 / 2$, without the need for oversampling of the received signal. In addition, the algorithm converges extremely fast and yields accurate estimation of frequency offset within $15-20$ time instants with only 50 particles.

The remainder of this paper is organised as follows. The underlying signal model is described in Section II and the particle filter formulation is given in Section III. The proposed particle filtering algorithm is described in Section IV. The simulation results are presented in Section V. Finally, conclusions are drawn in Section VI.

\section{SySTEM MODEL}

\section{A. Signal Model}

We consider a digital communication system where the transmitter sends the data symbols, chosen from a discrete alphabet of size $M$, over an Additive White Gaussian Noise (AWGN) channel. The noisy signal at the output of receiver 
matched filter can be written as

$$
z(t)=\sum_{d=0}^{D-1} x_{d} h(t-d T+\tau T) e^{j 2 \pi F t}+v(t),
$$

where $x_{d}$ is the $d$ th transmitted symbol, $D$ is the frame size, i.e., a transmission block of bits, $h(t)$ represents the overall pulse shaping filter response for transmitter and receiver ends, $T$ is the symbol period, $\tau$, is the normalised fractional unknown timing offset $\left(|\tau| \leq \frac{1}{2}\right)$ between the transmitter and receiver, $F$ is the frequency offset between the transmitter and receiver oscillators in $\mathrm{Hz}$ and $v$, with variance $\sigma_{v}^{2}$, is the AWGN.

The equivalent discrete-time system model after sampling is given by

$$
z_{k}=\sum_{d=0}^{D-1} x_{d} h\left(k T_{s}-d T+\tau T\right) e^{j 2 \pi f k}+v_{k},
$$

where $z_{k}=z\left(k T_{s}\right), v_{k}=v\left(k T_{s}\right), T_{s}=T / Q$ is the sampling period, $Q$ is the number of samples per symbol and $f=F / F_{s}$ is the digital frequency offset in cycles/sample. The frequency offset, $f$, has the range $\left[-F /\left(2 F_{s}\right), F /\left(2 F_{s}\right)\right]$ for the baseband signal model lying between $-F / 2$ and $F / 2 \mathrm{~Hz}$.

Assuming $h(t)$ to be a causal filter with finite duration $L$, the sampled received signal can be expressed as

$$
z_{k}=\sum_{d=k-L}^{k} x_{d} h\left(k T_{s}-d T+\tau T\right) e^{j 2 \pi f k}+v_{k},
$$

The above equation can also be written in vector form as

$$
z_{k}=\mathbf{x}_{k}^{T} \mathbf{h}_{k}(\tau) e^{j 2 \pi f k}+v_{k},
$$

where the superscript $(\cdot)^{T}$ denotes the hermitian transpose operator, $k=0,1, \ldots, D-1, \mathbf{x}_{k}=\left[x_{k-L}, \ldots, x_{k}\right]^{T}$ and $\mathbf{h}_{k}(\tau)=[h(L T+\tau), \ldots, h(\tau)]^{T}$ are $(L+1) \times 1$ vectors.

In this paper, we assume that the timing offset is perfectly compensated, i.e., $\mathbf{h}_{k}(\tau)=[0, \ldots, 0,1]^{T}$ with $L$ zeros in the pre-cursor. In addition, we are able to demonstrate good results without oversampling, $Q=1$ implying $F_{s}=F$, and for the full range for the frequency offset $(-0.5,0.5)$. Hence we focus on the problem of jointly estimating the carrier frequency offset and data symbols. Hence, (4) simplifies to scalar form

$$
z_{k}=x_{k} e^{j 2 \pi f k}+v_{k},
$$

\section{B. Estimation Objective}

The problem of joint blind estimation of transmitted symbols and the frequency offset, is to estimate data symbols $x_{0: D-1}=\left\{x_{0}, \ldots, x_{D-1}\right\}$ and corresponding carrier frequency offset $f_{0: D-1}$, from the available observations $z_{0: D-1}=\left\{z_{0}, \ldots, z_{D-1}\right\}$. Note that the notation $f_{0: k}$ denotes the estimates of $f$ at time instants 0 to $k$ and it does not represent a time-varying frequency offset.

From a Bayesian perspective, all the necessary information for the estimation of unknown parameters $\left(x_{k}, f_{k}\right)$ at time $k$, is contained in the joint posterior probability distribution function $p\left(x_{0: k}, f_{0: k} \mid z_{0: k}\right)$. Since this distribution is analytically intractable, we use a particle filter to represent the posterior pdf by means of a discrete probability measure with random support.

\section{PARTICLE Filter Formulation}

In this section, we formulate a particle filter based method for approximating $p\left(x_{0: k}, f_{0: k} \mid z_{0: k}\right)$. To this aim, we first introduce a change of frequency variable as $\mathfrak{f}=f+0.5$ such that $\mathfrak{f}$ has the range $[0,1]$. The reason for this change of variable is explained in Section IV-B.

The particle filter approximates the posterior pdf of unknown variables, $p\left(x_{0: k}, \mathfrak{f}_{0: k} \mid z_{0: k}\right)$, by a set of $N$ particles, $\left\{\left(x_{0: k}, \mathfrak{f}_{0: k}\right)^{(i)}\right\}_{i=1}^{N}$, with associated weights $\left\{w_{k}^{(i)}\right\}_{i=1}^{N}$. One of the most widely used techniques for implementing particle filters and calculating the weights is the Sequential Importance Sampling (SIS) method. The basic principle of this technique is to build a recursive empirical approximation of a desired PDF by drawing samples from a different distribution (called the importance function) and assigning appropriate normalised importance weights to these samples. The reader is referred to [12] for a tutorial introduction to this technique.

According to this technique, the weights can be computed as

$$
w_{k}^{(i)} \propto \frac{p\left(x_{0: k}^{(i)}, \mathfrak{f}_{0: k}^{(i)} \mid z_{0: k}\right)}{\pi\left(x_{0: k}^{(i)}, \mathfrak{f}_{0: k}^{(i)} \mid z_{0: k}\right)}
$$

where the importance function $\pi(\cdot)$ is chosen to admit a factorization of the form

$$
\begin{aligned}
\pi\left(x_{0: k}^{(i)}, \mathfrak{f}_{0: k}^{(i)} \mid z_{0: k}\right)=p\left(\mathfrak{f}_{k} \mid \mathfrak{f}_{0: k-1}^{(i)}, x_{0: k-1}^{(i)}, z_{0: k-1}\right) \\
\quad \times p\left(x_{k} \mid x_{0: k-1}^{(i)}, \mathfrak{f}_{0: k}^{(i)}, z_{0: k}\right)
\end{aligned}
$$

By resorting to a recursive decomposition of the posterior distribution, the numerator in (6) can be expressed as

$$
\begin{aligned}
p\left(x_{0: k}^{(i)}, \mathfrak{f}_{0: k}^{(i)} \mid z_{0: k}\right) \propto p\left(\mathfrak{f}_{k} \mid \mathfrak{f}_{0: k-1}^{(i)}, x_{0: k-1}^{(i)}, z_{0: k-1}\right) \\
\times p\left(z_{k} \mid x_{k-1}^{(i)}, \mathfrak{f}_{k}^{(i)}\right) \times p\left(x_{k} \mid x_{0: k-1}^{(i)}, \mathfrak{f}_{0: k}^{(i)}, z_{0: k}\right) \\
\times p\left(x_{0: k-1}^{(i)}, \mathfrak{f}_{0: k-1}^{(i)} \mid z_{0: k-1}\right)
\end{aligned}
$$

We can identify that two PDFs in (8) are the same as in (7). Substituting (8) and (7) in (6) and after some manipulation, we obtain

$$
w_{k}^{(i)}=w_{k-1}^{(i)} p\left(z_{k} \mid x_{k-1}^{(i)}, \mathfrak{f}_{k}^{(i)}\right)
$$

The posterior PDF can be then approximated as

$$
p\left(x_{0: k}, \mathfrak{f}_{0: k} \mid z_{0: k}\right) \approx \sum_{i=1}^{N} w_{k}^{(i)} \delta\left(\left(x_{0: k}, \mathfrak{f}_{0: k}\right)^{(i)}-\left(x_{0: k}, \mathfrak{f}_{0: k}\right)\right)
$$

where $\delta(\cdot)$ denotes Dirac's delta function. Using this approximated posterior PDF, estimates of data and frequency offset can be easily computed. 


\section{Proposed Algorithm for Data Detection And FREQUENCY OFFSET ESTIMATION}

In this section, based on the particle filter formulation presented in Section III, we describe the proposed algorithm for joint data detection and frequency offset estimation. The algorithm consists of the following steps:

\section{A. Initialization}

We assume that the prior distribution of the transmitted symbol and frequency offset, $p\left(x_{-1}, \mathfrak{f}_{-1}\right)$, is known. The density of the frequency offset, $\mathfrak{f}_{-1}$, is considered to be uniform in the range $(0,1)$, since the applied frequency offset, $f$, is uniformly distributed in the range $\left(-\frac{1}{2}, \frac{1}{2}\right)$. In addition, we assume that the first symbol, $x_{-1}$, is known. This simple assumption helps us to avoid the $M$-fold ambiguity of the constellation and to acquire the full-range acquisition of the frequency offset. Note that the use of a single pilot bit transmission to resolve ambiguities is common to many blind schemes [13]. Thus, we initialize the algorithm at time $k=-1$ as $x_{-1}^{(i)}=x_{-1}$ and $\mathfrak{f}_{-1}^{(i)} \sim \mathcal{U}(0,1), i=1,2, \ldots, N$. We initialize the weights of all the particles to be equal, i.e., $w_{-1}^{(i)}=1 / N$.

\section{B. Importance Sampling}

The sampling from the importance function in (7) is accomplished in two steps. First, we obtain the frequency offset sample from $p\left(\mathfrak{f}_{k} \mid \mathfrak{f}_{0: k-1}^{(i)}, x_{0: k-1}^{(i)}, z_{0: k-1}\right)$. Since this density cannot be exactly determined, we must approximate it using a suitable density. Following the approach in [10], we approximate this distribution by a beta distribution. Since beta distribution has a range $[0,1]$, we introduced a change in the frequency variable in Section III to have the same range. Thus, we draw the frequency offset sample from

$$
\mathfrak{f}_{k} \sim \beta\left(\mathfrak{f}_{k} ; U_{k}, V_{k}\right)
$$

where beta distribution parameters $U_{k}$ and $V_{k}$, see [10], are obtained by

$$
\begin{gathered}
U_{k}=\overline{\mathfrak{f}}_{k}\left(\frac{\overline{\mathfrak{f}}_{k}\left(1-\overline{\mathfrak{f}}_{k}\right)}{\sigma_{\mathfrak{f}_{k}}^{2}}-1\right) \\
V_{k}=\left(1-\overline{\mathfrak{f}}_{k}\right)\left(\frac{\overline{\mathfrak{f}}_{k}\left(1-\overline{\mathfrak{f}}_{k}\right)}{\sigma_{\mathfrak{f}_{k}}^{2}}-1\right)
\end{gathered}
$$

where $\overline{\mathfrak{f}}_{k}$ and $\sigma_{\mathfrak{f}_{k}}^{2}$ can be computed as

$$
\begin{gathered}
\overline{\mathfrak{f}}_{k}=\sum_{i=1}^{N} w_{k-1}^{(i)} \mathfrak{f}_{k-1}^{(i)} \\
\sigma_{\mathfrak{f}_{k}}^{2}=\sum_{i=1}^{N} w_{k-1}^{(i)}\left(\mathfrak{f}_{k-1}^{(i)}-\overline{\mathfrak{f}}_{k}\right)^{2}
\end{gathered}
$$

After drawing a carrier frequency offset sample, we draw a sample of the transmitted symbols from the second term of the proposal distribution $p\left(x_{k} \mid x_{k-1}^{(i)}, \mathfrak{f}_{k}^{(i)}, z_{k}\right)$. Since the transmitted symbols are independent and identically distributed (i.i.d.) discrete uniform random variables, we can ignore the dependency on all of the previous time states and the sampling density can be rewritten as

$$
\begin{aligned}
p\left(x_{k}=X_{m} \mid x_{k-1}^{(i)}, \mathfrak{f}_{k}^{(i)}, z_{k}\right) & \propto p\left(z_{k} \mid x_{k}=X_{m}, x_{k-1}^{(i)}, \mathfrak{f}_{k}^{(i)}\right) \\
& =\mathcal{N}\left(\mu_{k}^{(i)}\left(X_{m}\right), \sigma_{v}^{2}\right)
\end{aligned}
$$

where $\mathcal{N}\left(\mu, \sigma^{2}\right)$ denotes the Gaussian distribution with mean $\mu$ and variance $\sigma^{2}, X_{m}$ is one of the possible transmitted symbols of the modulation alphabet, $\mathcal{X}=\left\{X_{1}, X_{2}, \ldots, X_{M}\right\}$ and $\mu_{k}^{(i)}\left(X_{m}\right)=X_{m} e^{j 2 \pi f_{k}^{(i)} k}$, where $f_{k}^{(i)}=\mathfrak{f}_{k}^{(i)}-0.5$. From (16), we can derive the probability mass function for each symbol of $\mathcal{X}$ as

$$
\begin{aligned}
\rho^{(i)}\left(x_{k}\right) & =p\left(x_{k}=X_{m} \mid x_{k-1}^{(i)}, \mathfrak{f}_{k}^{(i)}, z_{k}\right) \\
& =\frac{\mathcal{N}\left(\mu_{k}^{(i)}\left(X_{m}\right), \sigma_{v}^{2}\right)}{\sum_{X \in \mathcal{X}} \mathcal{N}\left(\mu_{k}^{(i)}(X), \sigma_{v}^{2}\right)}
\end{aligned}
$$

where $i=1,2, \ldots, N$. Hence, we draw the transmitted symbol according to

$$
x_{k}^{(i)} \sim \rho^{(i)}\left(x_{k}\right)
$$

\section{Weight update}

After obtaining the new particles, we update their corresponding importance weights. Since all the possible transmitted symbols are equiprobable, we can rewrite the weight update expression (9) as

$$
\begin{aligned}
\tilde{w}_{k}^{(i)} & \propto w_{k-1}^{(i)} \sum_{X \in \mathcal{X}} p\left(z_{k} \mid x_{k}=X, x_{k-1}^{(i)}, \mathfrak{f}_{k}^{(i)}\right) \\
& =w_{k-1}^{(i)} \sum_{X \in \mathcal{X}} \mathcal{N}\left(\mu_{k}^{(i)}(X), \sigma_{v}^{2}\right)
\end{aligned}
$$

where $\tilde{w}_{k}^{(i)}$ is the non-normalized importance weight for the $i$ th particle and we use the assumption that $p\left(x_{k} \mid x_{k-1}^{(i)}\right)=p\left(x_{k}\right)$. Finally, we normalize the weights as

$$
w_{k}^{(i)}=\frac{\tilde{w}_{k}^{(i)}}{\sum_{n=1}^{N} \tilde{w}_{k}^{(n)}}
$$

\section{Resampling}

A well known problem in the practical implementation of the Sequential Importance Sampling algorithm is that the discrete measure of the particles $\left\{\left(x_{k}, \mathfrak{f}_{k}\right)^{(i)}\right\}$ degenerates quickly, i.e., after a few time steps, most of the importance weights have negligible values $\left(w_{k}^{(i)} \simeq 0\right)$. The common solution to this problem is to resample the particles [12]. Resampling eliminates the particles with small weights, while replicating those with larger weights. Thus the basic resampling operation involves generation of $N$ new particles by sampling the discrete set $\left\{\left(x_{k}, \mathfrak{f}_{k}\right)^{(i)}\right\}_{i=1}^{N}$ with probabilities $w_{k}^{(i)}$ and then resetting the importance weights to equal values $1 / N$. Resampling at every time step is not needed [12]. We consider that resampling is carried out in our algorithm whenever the effective sample size of the particle filter, $N_{\text {eff }}$, approximated as,

$$
N_{\text {eff }}=\frac{1}{\sum_{i=1}^{N}\left(w_{k}^{(i)}\right)^{2}} \leq N / 2
$$


goes below a certain threshold (typically a fraction of $N$ ) [14].

In addition to the standard resampling as described above, we propose two new resampling schemes to take into account carrier offset estimation ambiguity at the edge of the frequency range and for fine tuning the estimates. These are described in the next two subsections. It must be noted that without these two steps, the algorithm may not always lead to meaningful results.

\section{E. Resampling Guidelines}

In this paper, we consider the full frequency offset acquisition range $(-0.5,0.5)$ as a cyclic range. When the number of particles is small, the particle filter may converge to a value which is close to but not the true carrier frequency offset estimate. Since even small residual errors in CFO estimation can lead to large errors in data detection, we propose a check on the weights for fine tuning the estimates. Once the weights fall below a selected threshold, indicating convergence, we regenerate the particles in the range \pm 0.015 of the most recurring particle and update the corresponding weights. In addition, the edges of the frequency offset range, $(-0.5,-0.45)$ and $(0.45,0.5)$, are very sensitive to error since even a small noise can perturb the received signal and lead to the posterior density of frequency offset to evolve in the wrong estimation region. We mitigate this problem by putting a check on the first symbol after resampling. The results in Sec. V-A indicate that with this resampling, the algorithm achieves very accurate estimates of the carrier frequency offset.

\section{F. Estimation}

Finally, the importance weights and the drawn samples for the carrier frequency offset are used to compute the Minimum Mean Square Error (MMSE) estimate of the true frequency offset $\hat{f}_{k}$ as

$$
\hat{f}_{k}=\left(\sum_{i=1}^{N} \mathfrak{f}_{k}^{(i)} w_{k}^{(i)}\right)-0.5
$$

In addition, we compute the maximum a posteriori (MAP) estimates of the $k^{\text {th }}$ transmitted symbol as

$$
\hat{x}_{k}=\arg \max _{X \in \mathcal{X}}\left\{\sum_{i=1}^{N} w_{k}^{(i)} \delta\left(x_{k}^{(i)}-X\right)\right\}
$$

The proposed algorithm is summarized in Fig. 1.

\section{Simulation Results}

In this section, we present simulation results to verify the performance of our proposed algorithm. We consider a communication system with a Binary Phase Shift Keying (BPSK) modulation and an AWGN channel. Note that AWGN channel is considered by many authors in the synchronization context [15], [16] . The random frequency offset is assumed to be uniformly distributed in the range $(-0.48,0.48)$, i.e., approximately full acquisition range acquisition. We use $N=$ 50,100 and 200 particles to estimate data symbols and the frequency offset jointly. The simulation results are averaged over $R=600$ Monte Carlo simulations, with each run consisting of a block of $M=100$ transmitted bits.

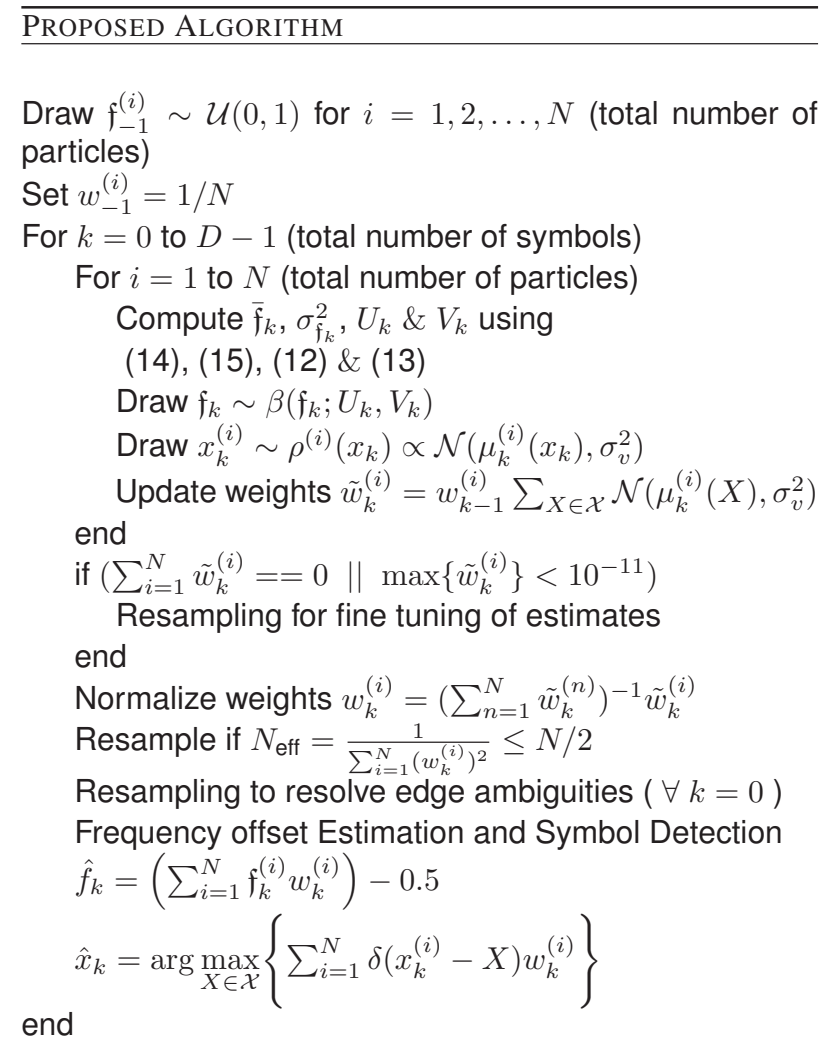

Fig. 1. Proposed Algorithm for Joint Blind Carrier Frequency Offset Estimation and Data Detection.

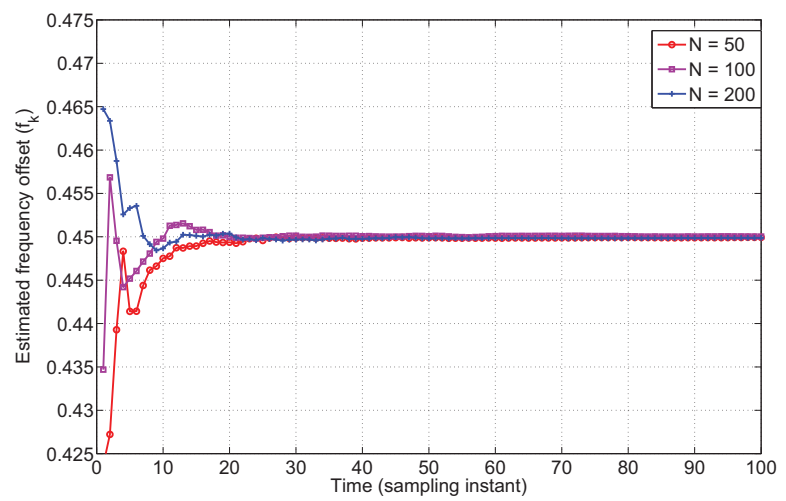

Fig. 2. Estimate of the frequency offset $\hat{f}_{k}$ at different time instants for SNR $=14 \mathrm{~dB}$, system frequency offset, $f=0.45$ and number of particles $N=50,100$ and 200 , respectively.

\section{A. Acquisition range and fast convergence}

Fig. 2 shows one realization of the particle filter estimates of the frequency offset at different time instants for a Signal to Noise Ratio (SNR) of $14 \mathrm{~dB}$. The true frequency offset is $f=$ 0.45 or equivalently $\mathfrak{f}_{k}=0.95$. The figure demonstrates that the estimated frequency offset $\hat{f}_{k}$ converges to the true value after just $15-20$ time instants, e.g., for $N=50$ particles, the value is $\hat{f}_{k}=0.4494$ after 20 time instants which is very close to the true estimate. In addition, as we increase the number of particles, the estimate converges more rapidly. 


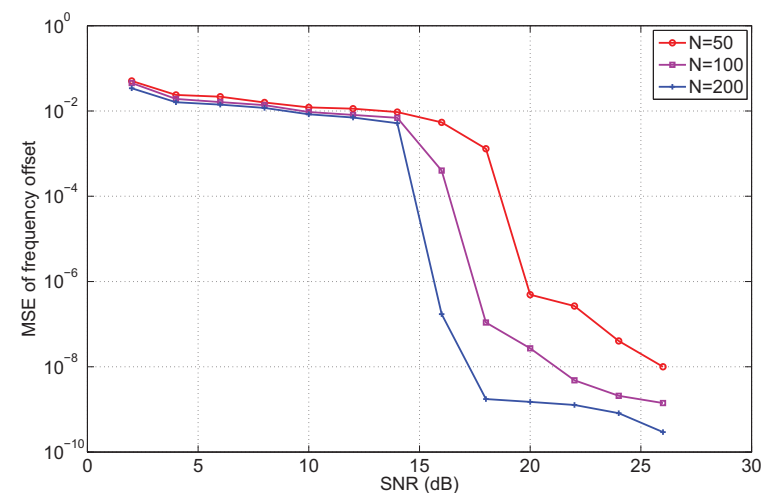

Fig. 3. MSE of frequency offset estimation as a function of SNR (dB), for different number of particles $N=50,100$ and 200 .

\section{B. Mean Square Error (MSE) of frequency offset estimation}

Fig. 3 shows the Mean Square Error (MSE) of frequency offset estimation for different values of SNR. The MSE was approximated as, MSE $=\sum_{\ell=1}^{R}\left(f(\ell)-\hat{f}_{D-1}(\ell)\right)^{2}$, where $R$ is the number of Monte Carlo simulation runs for each value of SNR, $f(\ell)$ is the introduced random frequency offset for $\ell$ th simulation and $\hat{f}_{D-1}(\ell)$ is the estimate of frequency offset for $(D-1)$ th symbol and $\ell$ th simulation. We can see that the MSE decreases considerably after $10 \mathrm{~dB}$ SNR and increasing the number of particles results in lower MSE value.

\section{Bit Error Rate (BER) performance}

Fig. 4 show the BER as a function of the SNR. The reference curve in this figure is the theoretical BER curve for BPSK in AWGN channels with no frequency offset error. We can see that there is a performance loss compared to the reference curve, e.g., an SNR of $16 \mathrm{~dB}$ is required to achieve a BER of $10^{-5}$ for $N=200$ particles which is approximately $6 \mathrm{~dB}$ from the reference curve. However we can see that at high SNR, the slope of the simulated BER curves approaches that of the reference curve which confirms the correct working of the proposed algorithm.

The apparent loss in performance can be attributed to the fact that the carrier frequency offset is a very sensitive synchronization parameter. Even small residual errors in frequency offset estimation (of the order of 0.01) can lead to huge errors in data detection and bias the averaged BER results. The presented BER simulation results are unbiased results, i.e., we have not artificially discarded any initial bits to allow the algorithm to converge or $5 \%$ extreme results as suggested in [9]. Also note that the BER result in Fig. 4 corresponds to the worst case scenario with frequency offset randomly distributed in the full acquisition range.

\section{CONCLUSIONS}

In this paper, we have developed a particle filter algorithm to jointly estimate the carrier frequency offset and transmitted data. We have proposed two new resampling procedures to deal with carrier offset estimation ambiguity at the edges and to fine tuning estimates. The simulation results confirmed that the proposed algorithm achieves full-range acquisition of

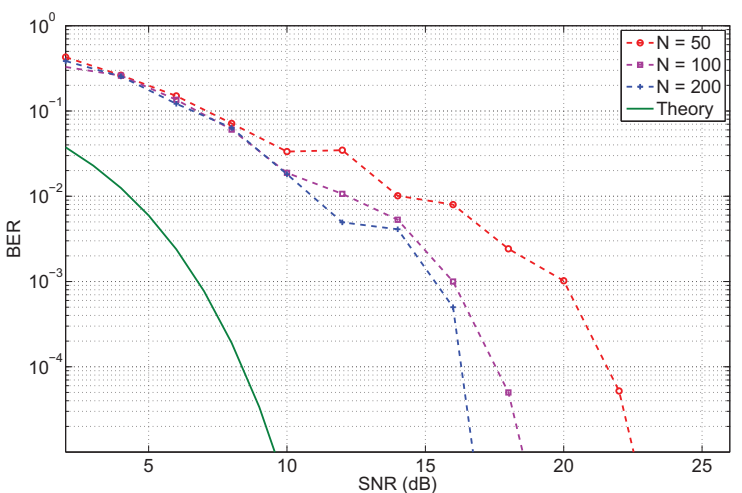

Fig. 4. BER of the particle filter receiver as a function of SNR (dB), for different number of particles $N=50,100$ and 200 .

frequency offset and achieves fast accurate convergence in about 15-20 time instants with only 50 particles.

\section{REFERENCES}

[1] C. Y. Chi, C. C. Feng, C. H. Chen, and C. Y. Chen, Blind Equalization and System Identification. Springer, 2006.

[2] M. Morelli and U. Mengali, "Carrier-frequency estimation for transmissions over selective channels," IEEE Trans. Commun., vol. 48, no. 9 , pp. 1580-1589, Sep 2000.

[3] U. Mengali and A. N. Andrea, Synchronization Techniques for Digital Receivers. Plenum Press, New York, 1997.

[4] Y. Yu, A. P. Petropulu, H. V. Poor, and V. Koivunen, "Blind estimation of multiple carrier frequency offsets," in Proc. IEEE International Symposium on Personal, Indoor \& Mobile Radio Communications, 2007.

[5] F. Gini and G. B. Giannakis, "Frequency offset and symbol timing recovery in flat-fading channels: A cyclostationary approach," IEEE Trans. Commun., vol. 46, no. 3, pp. 400-411, Mar. 1998.

[6] Y. Wang, K. Shi, and E. Serpedin, "Non data-aided feedforward carrier frequency offset estimators for QAM constellations: A nonlinear leastsquares approach," EURASIP Journal on Applied Signal Processing, vol. 13, pp. 1993-2001, 2004.

[7] F. Daum, "Nonlinear filters: beyond the Kalman filter," IEEE Aerospace and Electronic Systems Magazine, vol. 20, no. 8, pp. 57-69, Aug. 2005.

[8] P. M. Djuric, J. Kotecha, J. Zhang, Y. Huang, T. Ghirmai, M. F. Bugallo, and J. Miguez, "Particle filtering," IEEE Signal Processing Magazine, vol. 20, no. 5, pp. 19-38, Sep 2003.

[9] C. J. Bordin and M. G. S. Bruno, "Particle filters for joint blind equalization and decoding in frequency-selective channels," IEEE Transactions on Signal Processing, vol. 56, no. 6, pp. 2395-2405, June 2008.

[10] T. Ghirmai, "Sequential Monte Carlo method for fixed symbol timing estimation and data detection," in Proc. IEEE Information Sciences and Systems, 2006.

[11] T. Ghirmai, N. F. Bugallo, J. Miguez, and P. M. Djuri, "A sequential monte carlo method for adaptive blind timing estimation and data detection," IEEE Transactions on Signal Processing, vol. 53, no. 8, pp. 2855-2865, Aug. 2005.

[12] A. Doucet, S. Godsill, and C. Andrieu, "On sequential Monte Carlo sampling methods for bayesian filtering," Statistics and Computing, vol. 10, pp. 197-208, 2000.

[13] E. Beres and R. Adve, "Blind channel estimation for orthogonal STBC in MISO systems," IEEE Trans. Veh. Technol., vol. 56, no. 4, pp. 2042 2050, Jul. 2007.

[14] M. S. Arulampalam, S. Maskell, N. Gordon, and T. Clapp, "A tutorial on particle filters for online nonlinear/non-gaussian Bayesian tracking," IEEE Trans. Signal Process., vol. 50, no. 2, pp. 174-188, Feb. 2002.

[15] J. Gunther and T. Moon, "Burst mode synchronization of QPSK on AWGN channels using kurtosis," IEEE Trans. Commun., vol. 57, no. 8, pp. 2453-2462, Aug. 2009.

[16] J. T. Yuan and Y. F. Huang, "Blind carrier phase acquisition and tracking for 8-VSB signals," IEEE Trans. Commun., vol. 58, no. 3, pp. 769-774, Mar. 2010. 